\title{
An Electron Microscope Study of the Mesosomes of a Penicillinase-producing Staphylococcus
}

\author{
By C. D. BEATON \\ Commonwealth Serum Laboratories, Parkville, Victoria, 3052, Australia
}

(Accepted for publication 29 June 1967 )

SUMMARY

Thin sections of Staphylococcus aureus were examined in the electron microscope, before and after treatments which released part of their cell-bound penicillinase. In all cases, such treatment resulted in alteration of the mesosome structure: the mesosome was replaced by a series of pockets or invaginations in the cytoplasmic membrane. The converse situation was not always true; cocci treated in such a way as to alter the mesosome structure did not necessarily release penicillinase. This leads to the conclusion that structural alteration of the mesosome is only one of a number of steps in the release of penicillinase.

\section{INTRODUCTION}

When penicillinase is synthesized by Bacillus and Staphylococcus, only part of the total penicillinase produced is released into the medium as an exo-enzyme, the rest remaining firmly bound to the cell. A mechanism for this synthesis and the subsequent release of penicillinase in Bacillus was proposed by Lampen (1965), based on the assumption that the mesosome-the membranous intracytoplasmic organelle found in many bacteria-is the site of synthesis of cytoplasmic membrane and of penicillinase. Thus it appeared to be worthwhile, in an investigation of the release of penicillinase, to study the ultrastructure of the mesosomes. Suganuma (1963) studied the mesosomes of staphylococci and correlated alteration in mesosome morphology with age of culture; however, he did not state whether the strain he used produced penicillinase. Coles \& Gross $(1967 a, b)$ studied some of the factors influencing the release of penicillinase as an exo-enzyme, and described the release of substantial amounts of penicillinase which had previously been bound to the cell. It was decided to examine in the electron microscope thin sections of organisms treated by the methods of Coles \& Gross to determine whether the in vitro release of penicillinase caused any structural changes, particularly in the morphology of the mesosomes.

\section{METHODS}

Staphylococcus aureus strain 8325 $\alpha i^{-} p^{+}$(Novick \& Richmond, 1965) was grown overnight in the CY medium used by Novick (1963), and also in a medium modified by using $0.05 \mathrm{M}$-tris as buffer instead of the $\beta$-glycerophosphate specified by Novick; this modified medium will be referred to as tris $+C Y$ medium.

Organisms grown in both these media produced penicillinase, but whereas the CY medium resulted in about $45 \%$ of the total penicillinase being released to the 
growth medium in a soluble form, growth in the tris + CY medium resulted in less than $2 \%$ of the total penicillinase being released, the other $98 \%$ remaining firmly bound to the organism (Coles \& Gross, 1967a). Thus the tris + CY grown organisms were the better starting material for the in vitro study of penicillinase release, and samples of these organisms were subsequently incubated for $\mathrm{I} \mathrm{hr}$ at $37^{\circ}$ in a medium containing $0.15 \mathrm{M}-\mathrm{Na}_{2} \mathrm{HPO}_{4}$ and $0.0 \mathrm{I} \mathrm{M}$-glucose, adjusted to $\mathrm{pH} 7.6$. This treatment resulted in the release in a soluble form of $30 \%$ of the previously cell-bound penicillinase. Details of these procedures and the method for determining penicillinase are given in the papers of Coles \& Gross $(1967 a, b)$ in which the biochemical aspects of the penicillinase release are reported.

Samples of cocci were fixed by a modification of the method of Kellenberger, Ryter \& Séchaud (1958): they were prefixed by the addition of $10 \%(\mathrm{v} / \mathrm{v})$ of the Kellenberger fixative; after $30 \mathrm{~min}$. they were centrifuged down, and resuspended in the fullstrength fixative. After $2 \mathrm{hr}$, the cocci were again centrifuged down, then suspended in warm $I \%(w / v)$ agar and this suspension spread on a glass slide. After the agar had solidified, it was cut into small cubes which were fixed overnight. A $2 \mathrm{hr}$ treatment with uranyl acetate was used, and after ethanol dehydration the specimens were embedded in Araldite. Sections about $90 \mathrm{~m} \mu$ thick were cut by using an LKB Ultrotome, stained for $30 \mathrm{~min}$. with a $\mathrm{I} \%(\mathrm{w} / \mathrm{v})$ solution of uranyl acetate in ethanol, and examined in a Siemens Elmiskop I electron microscope.

\section{RESULTS}

\section{Staphylococci grown in $C Y$ or tris $+C Y$ medium}

There was little difference in morphology between cocci grown in CY medium and those grown in the tris $+\mathrm{CY}$ medium, apart from the easier visibility of the nuclear material in the CY-grown cocci. Since the cocci grown in tris $+\mathrm{CY}$ were used as the starting material in the study of the release of penicillinase, all micrographs of normal cocci shown are of organisms which had been grown in the tris + CY medium.

A conspicuous feature of the morphology of these cocci was the presence of mesosomes in large numbers and of various shapes and sizes. There appeared to be three types of mesosome: a lamellar type composed of concentric whorls of membrane, a vesicular type which was a collection of vesicles bounded by a membrane, and a mesosome which was a mixture of both types.

In Pl. I, fig. I, can be seen a coccus containing a mesosome of the lamellar type; the mesosome is bounded by an infolding of the cytoplasmic membrane. In the case of staphylococci prepared for electron microscopy as described above, the cytoplasmic membrane appears as a triple-layered membrane with a dark outer layer, a white gap, and a greyish inner layer. As would be expected, when this cytoplasmic membrane doubles back on itself, the bounding membrane of the mesosome then has a grey outer layer and a black inner layer.

In Pl. I, fig. 2, is a coccus containing a mesosome of the vesicular type; the vesicles appear to be rolled up portions of the mesosome membrane since they have a dark outer layer, a small gap, and contain greyish material, similar to the grey layer of membrane on the inside. Thus, it would seem that the vesicles result from a further invagination and closing off of the bounding membrane of the mesosome as Fitz-James (1960) suggested. 
In Pl. I, figs. 3, 4, can be seen cocci which contain mesosomes of the mixed type. In the organisms in both these figures, the mesosomes contain vesicles and show some evidence of the lamellar type of structure.

A spatial relationship exists between the mesosome and the developing cross-wall of Staphylococcus aureus; this is shown in Pl. 2, figs. 5-7, illustrating adjacent sections of the same coccus. Both mesosomes are seen to be in contact with the developing cross-wall. On the other hand, the set of adjacent sections shown in Pl. 2, figs. 8-IO, is of a coccus which contained a completed cross-wall and which also contained two mesosomes in each half of the coccus; three of these mesosomes opened directly into the space between the cell-wall and the cytoplasmic membrane, and so it would seem possible that these mesosomes are responsible for some function other than the synthesis of cell-wall material.

\section{Organisms grown in tris $+C Y$ medium and then incubated to release penicillinase}

When cocci grown in the tris $+\mathrm{CY}$ medium were incubated for $\mathrm{I} \mathrm{hr}$ in glucose + phosphate medium, about $30 \%$ of the previously cell-bound penicillinase was released in soluble form. Morphologically, what was seen in these cocci was the almost complete absence of mesosomes and the appearance of a new type of structure, which appeared to be an invagination of the cytoplasmic membrane, probably best described as a pocket.

Plate 3, fig. I I, shows a cell containing several of these pockets. It would appear that there was some relationship between these pockets and the mesosomes which were present in the cocci before enzyme release, since there were frequently vesicles within the pockets, and occasionally in the gap between the cell wall and the cytoplasmic membrane. Thus, a process whereby the mesosome contents were everted into the wall-membrane gap could be postulated, with the pockets resulting from the cytoplasmic membrane surrounding a vesicle which had been everted in this way.

There were large numbers of pockets in these cocci; this is shown in Pl. 3, figs. I2, 13, which were adjacent sections of the one coccus. If eversion of the vesicles occurred as proposed, then the number of pockets observed would be proportional to the total number of vesicles originally present in the coccus. Although it would seem from the micrographs already described that this process of penicillinase release was accompanied by a shrinkage of the cytoplasmic membrane (Pl. 3, figs. II-I3), this seems inconsistent with the proposed mechanism. No membrane shrinkage occurred in the organisms shown in serial section in Pl. 4, figs. 14-16; the shrinkage observed in the other micrographs of the treated cocci might be an artifact, reflecting a greater osmotic sensitivity of these cocci.

One of the rare instances of a mesosome remaining after treatment to release penicillinase is shown in the portion of the coccus on the right of Pl. 4, fig. I5.

\section{Other treatments of normal cocci}

Normal cocci were subjected to the following treatments (Coles \& Gross, $1967 a, b$ ) which caused penicillinase release and alteration of the mesosome structure: incubation in $0.15 \mathrm{M}$-phosphate or arsenate for $\mathrm{I} \mathrm{hr}$ at $\mathrm{pH} 7.6$, which caused pocket formation; and incubation with $0.01 \mathrm{M}$ or $0.15 \mathrm{M}$-sodium citrate for $\mathrm{I} \mathrm{hr}$, which produced pocket formation and some retention of the original mesosome structure. 
Although $0.15 \mathrm{M}$-phosphate or arsenate at $\mathrm{pH} 6.0$ or 7.6 caused instantaneous pocket formation, at $\mathrm{pH} 6.0$, no penicillinase was liberated by phosphate. On the other hand, treatment with dextran sulphate (Coles \& Gross, 1967a) which, caused instantaneous liberation of surface-located penicillinase, produced no detectable change in mesosome structure. Thus alteration of the mesosome structure was always observed and might be a prerequisite for the release of penicillinase from within the organism. However, penicillinase release did not always follow such alteration of structure, as shown by the case of phosphate at o min. and $\mathrm{pH} 6 \cdot 0$. Other factors, therefore, appeared to be necessary to release penicillinase; alteration of mesosome structure may be an early step in this sequence.

\section{DISCUSSION}

There has been much speculation about the function of bacterial mesosomes since they were first discovered. Fitz-James (1960), in his study of spore formation in Bacillus, remarked on the function of the mesosomes and suggested that they were involved in the formation of transverse septa, and the laying down of membrane. Fitz-James also observed vesicles within the mesosomes and he suggested that they were formed by an invagination of the mesosome membrane. It is possible that this is also the case with the Staphylococcus mesosomes (Pl. I, figs. 3, 4) and that thinner sections would show this relationship more clearly.

Ryter \& Jacob (1964) have shown that there is a spatial relationship between the mesosomes and the nuclei and cross-walls of dividing Bacillus subtilis organisms. A similar spatial relationship between mesosomes, nuclei and cross-walls is seen in Staphylococcus aureus (P1. 2, figs. 5-7). It is possible that this spatial relationship reflects a functional relationship and that it demonstrates the mesosomes functioning as sites of synthesis of cross-wall material as suggested by Fitz-James (I960). However Pl. 2, figs. 8-ro, shows mesosomes with a direct connexion into the wall-membrane gap, in an organism with completed cross-walls. In such a case it would seem that the mesosomes are responsible for some function other than the synthesis of cross-wall material; this function could be the synthesis of other cellular materials such as penicillinase.

Lampen (1965) proposed that the mesosome is an essential element in the secretion of penicillinase by Bacillus. He suggested that penicillinase is inserted into and bound to the cytoplasmic membrane at its point of growth in the mesosome, and that release of this penicillinase is dependent on the membrane growing out of the mesosome. The findings reported here for Staphylococcus aureus are consistent with Lampen's hypothesis if it is assumed that vesicles are the sites of growth of new membrane, so that penicillinase is likewise bound to the vesicles; eversion of the vesicles to the wall-membrane space would then make this penicillinase accessible for release.

Eversion of vesicles was demonstrated by Fitz-James (1964) and Weibull (1965) in Bacillus megaterium, and by Ryter \& Jacob (1964) in B. subtilis. These authors used solutions containing sucrose to induce plasmolysis of the organisms; the 'osmotic buffer' used by Fitz-James contained $0.3 \mathrm{M}$-sucrose, Weibull used sucrose solutions of at least molar concentration for plasmolysis to occur, and Ryter and Jacob used $0.5 \mathrm{M}$-sucrose. The glucose + phosphate medium used in the present work contained 
Journal of General Microbiology, Vol. 50, No. I

Plate I
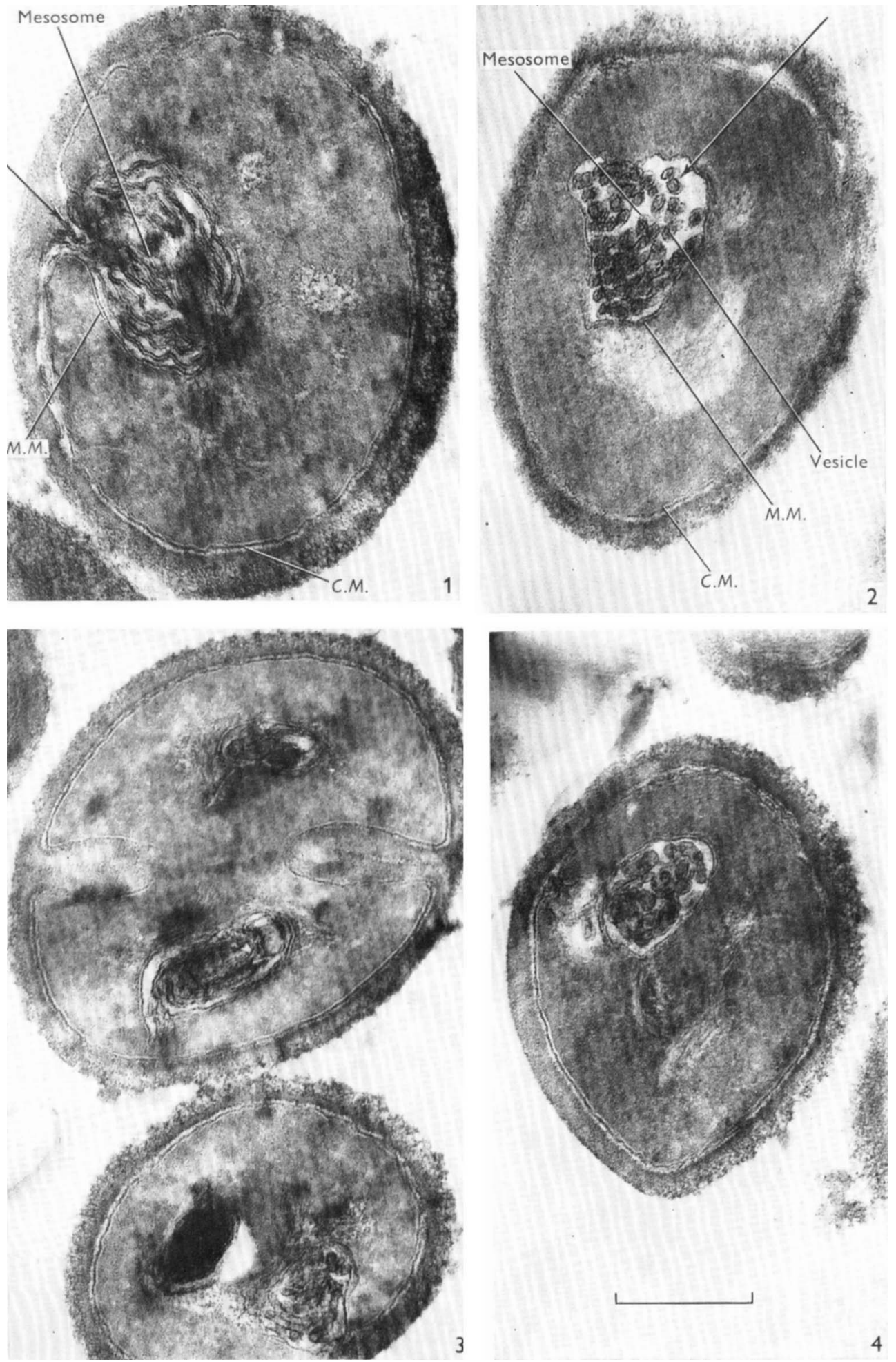

C. D. BEATON 
Journal of General Microbiology, Vol. 50, No. I

Plate 2
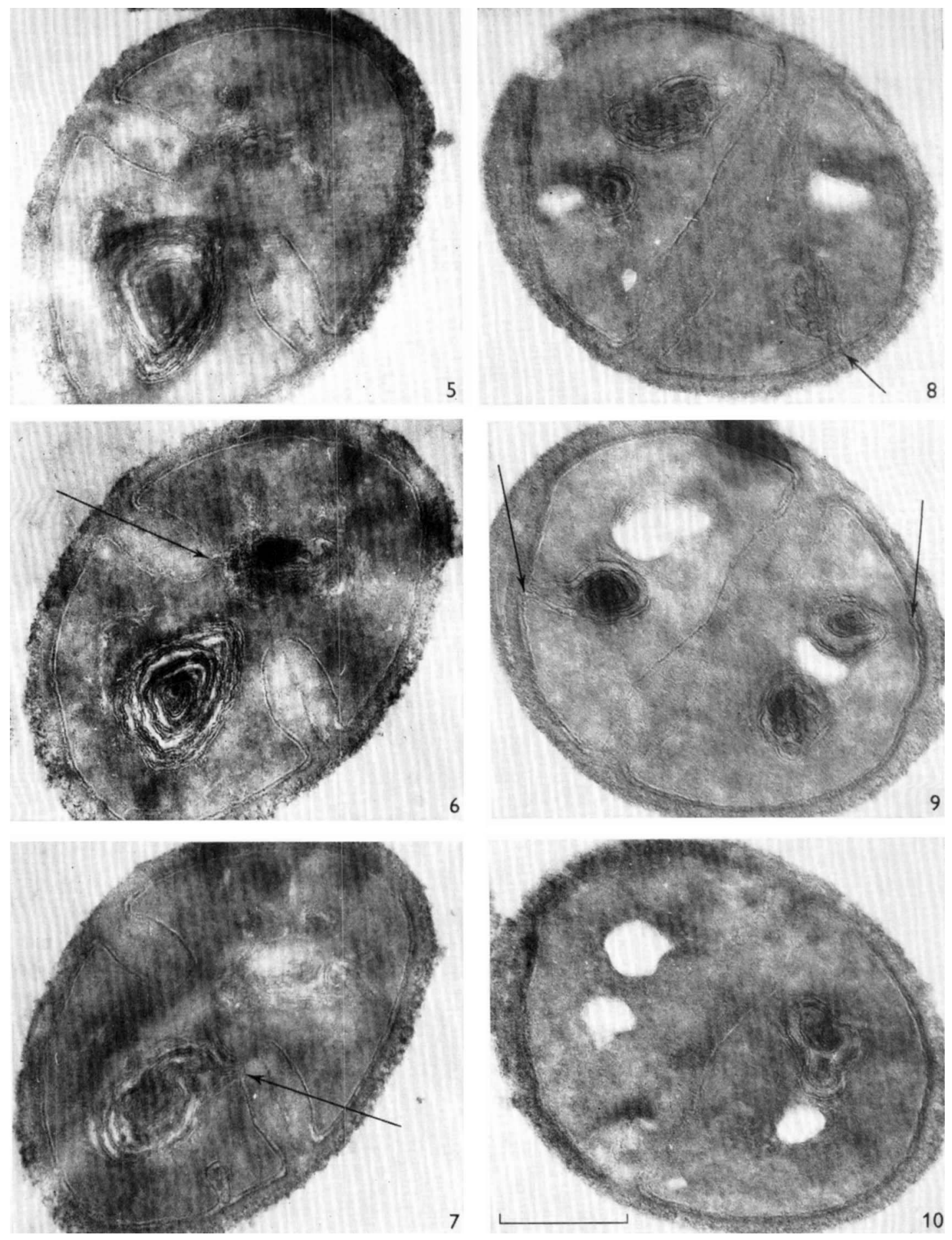

C. D. BEATON 
Journal of General Microbiology, Vol. 50, No. I

Plate 3
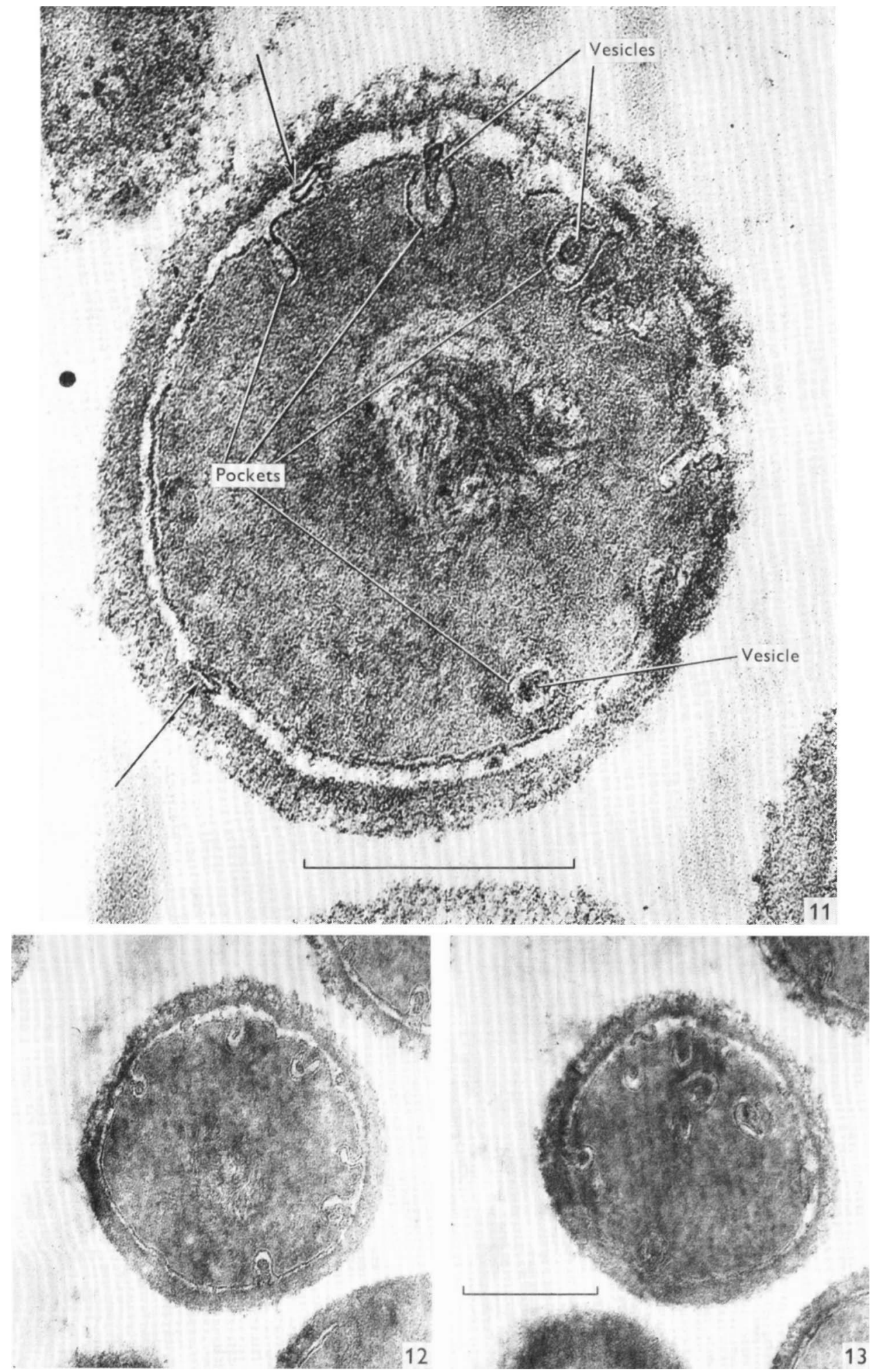

C. D. BEATON 
Journal of General Microbiology, Vol. 50, No. I

Plate 4
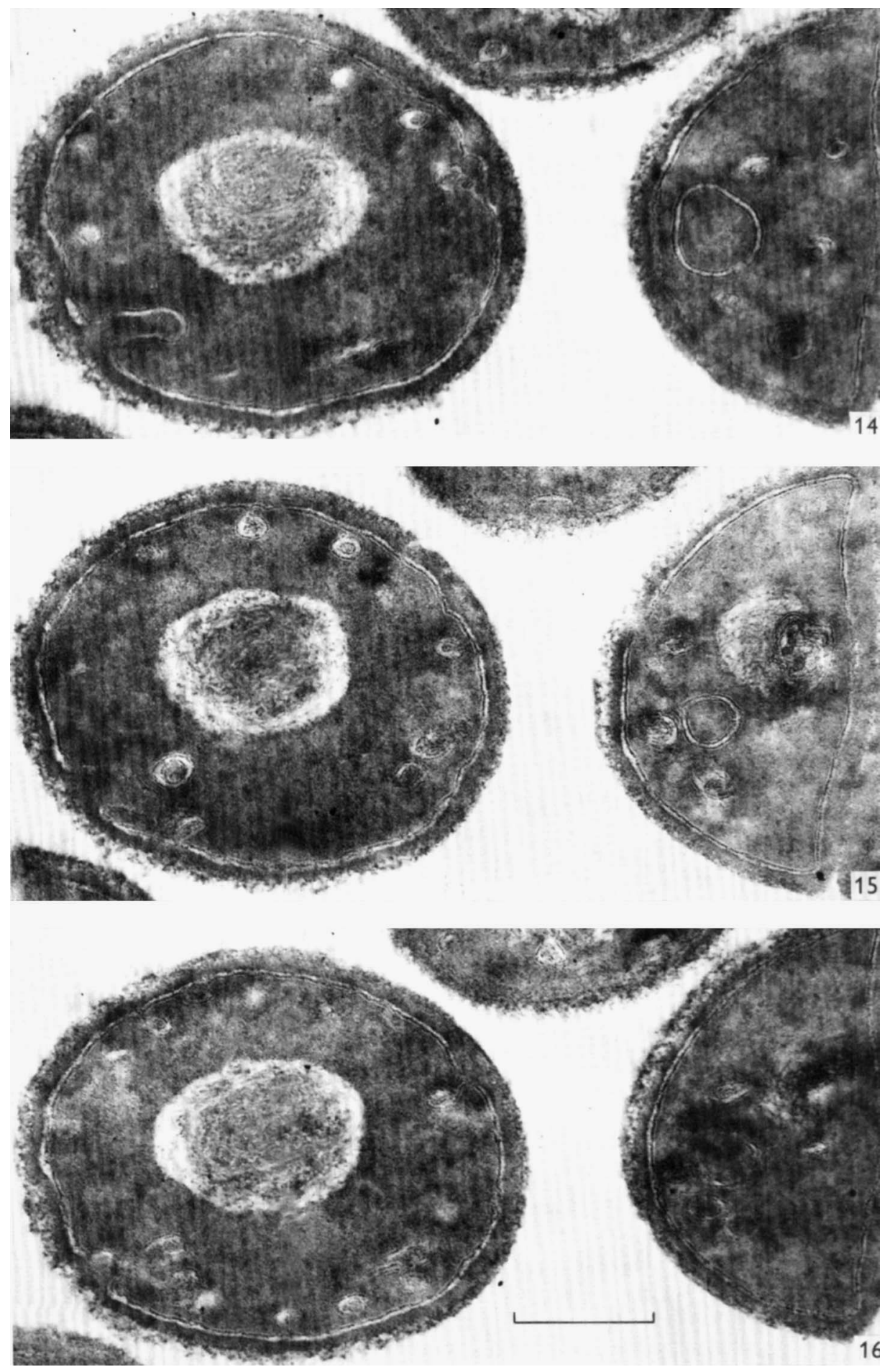

C. D. BEATON 
$0.0 \mathrm{I} \mathrm{M}$-glucose and $0.15 \mathrm{M}-\mathrm{Na}_{2} \mathrm{HPO}_{4}$ - concentrations too low to cause plasmolysis and thus ruling this out as an explanation for the observed vesicle eversion.

Therefore, although there does not appear to be a direct causal relationship between vesicle eversion and penicillinase release, the results presented here do indicate that the eversion of vesicles may be an early step in the release of penicillinase.

The author thanks Dr R. Gross and Dr N. W. Coles of these Laboratories for supplying the cultures used in this study and for much helpful discussion; he is grateful to Miss F. L. Kirby for technical assistance.

\section{REFERENCES}

Coles, N. W. \& Gross, R. (1967a). Liberation of surface-located penicillinase from Staphylococcus aureus. Biochem. J. 102, 742 .

Coles, N. W. \& Gross, R. (1967b). Influence of organic anions on the liberation of penicillinase from Staphylococcus aureus. Biochem. J. 102, 748.

Fitz-JAmes, P. C. (1960). Participation of the cytoplasmic membrane in the growth and spore formation of bacilli. J. biophys. biochem. Cytol. 8, 507.

Fitz-JAMES, P. C. (1964). Fate of the mesosomes of Bacillus megaterium during protoplasting. J. Bact. 87, 1483 .

KELlENBERGER, E., RYTER, A. \& SÉchAUd, J. (I958). Electron microscope study of DNA-containing plasms. II. Vegetative and mature phage DNA as compared with normal bacterial nucleoids in different physiological states. J. biophys. biochem. Cytol. 4, 671.

LAMPEN, J. O. (1965). Secretion of enzymes by micro-organisms. Symp. Soc. gen. Microbiol. 15 , I 15.

Novick, R. P. (1963). Analysis by transduction of mutations affecting penicillinase formation in Staphylococcus aureus. J. gen. Microbiol. 33, 121.

Novick, R. P. \& Richmond, M. H. (1965). Nature and interactions of the genetic elements governing penicillinase synthesis in Staphylococcus aureus. J. Bact. 90, 467.

RytER, A. \& JACOB, F. (1964). Étude au microscope électronique de la liasion entre noyau et mésosome chez Bacillus subtilis. Ann. Inst. Pasteur, Paris 107, 384.

Suganuma, A. (1963). Some features of membranous structures in Staphylococcus aureus. J. infect. Dis. 113, 179.

WeIBull, C. (1965). Plasmolysis in Bacillus megaterium. J. Bact. 89, I I5I.

\section{EXPLANATION OF PLATES}

On all micrographs, the bar represents $0.25 \mu$. The magnification is $\times 80,000$ for all micrographs except fig. II for which it is $\times 160,000$.

\section{Plate i. Normal cocci}

Fig. I. Section of a coccus illustrating the lamellar type of mesosome; the mesosome is composed of concentric whorls of membrane. The arrow indicates the point of attachment to the cytoplasmic membrane. The mesosome membrane (M.M.), and the cytoplasmic membrane (C.M.) are marked.

Fig. 2. An organism which illustrates the vesicular type of mesosome. The arrow indicates a vesicle in which the dark outer layer and the greyish inner layer of membrane can be seen.

Figs. 3, 4. Sections showing the mixed type of mesosome structure: the mesosomes show both lamellar and vesicular structure.

\section{Plate 2. Normal cocci}

Figs. 5-7. Adjacent sections of a dividing organism showing the attachment of mesosomes to the developing cross-walls.

Figs. 8-10. Adjacent sections of an organism which contained a completed cross-wall. The points of attachment of the mesosomes to the cytoplasmic membrane are shown. 
Plate 3. Cocci treated to release penicillinase

Fig. II. Section of an organism showing the pockets which appeared when penicillinase was released. Some of these pockets contain what appear to be vesicles. There is a small amount of membranous material (arrowed) in the space between the cell-wall and the cytoplasmic membrane.

Figs. 12, 13. Adjacent sections of one organism. This pair of micrographs gives some idea of the large number of pockets in each coccus.

Plate 4. Cocci treated to release penicillinase

Figs. I4-I6. Adjacent sections of two cocci, showing pocket formation. There has been negligible shrinkage of the cytoplasmic membrane in these organisms. 